\title{
Robust Beamforming for Enhancing Security in Multibeam Satellite Systems
}

\author{
Jian Zhang, Min Lin, Jian Ouyang, Wei-Ping Zhu, and Tomaso de Cola,
}

\begin{abstract}
This paper proposes a robust beamforming (BF) scheme to enhance physical layer security (PLS) of the downlink of a multibeam satellite system in the presence of either uncoordinated or coordinated eavesdroppers (Eves). Specifically, with knowing only the approximate locations of the Eves, we aim at maximizing the worst-case achievable secrecy rate (ASR) of the legitimate user (LU), subject to the constraints of per-antenna transmit power and quality of service (QoS) requirement of the LU. Since the optimization problem is non-convex, we first adopt the discretization method to deal with the unknown regions of the Eves and then exploit the log-sum-exp function to approximate the objective function. Afterwards, a BF method joint alternating direction method of multipliers (ADMM) with Dinkelbach iteration is presented to solve this non-convex problem. Finally, simulation results verify that our robust $\mathrm{BF}$ algorithm can effectively improve the security of multibeam satellite systems.
\end{abstract}

Index Terms

Multibeam satellite communications, robust beamforming, physical layer security, Alternating Direction Method of Multipliers

\section{INTRODUCTION}

$\mathbf{T}$ HANKS to the ability of offering high date rate transmission and achieving spectrum reuse, multibeam technology is considered as an indispensable element in the future satellite communication (SatCom) systems. [1]-[4]. However, the inherent nature of broadcast and wide coverage makes SatCom vulnerable to wiretapping. Therefore, security is regarded as a key issue in SatCom that has been widely studied in the open literatures. Although secure communication is traditionally achieved through cryptographic encryption at the upper layer, this approach faces great challenges with the emergence of new technologies such as cloud computing and quantum computing.

In recent years, physical layer security (PLS) technology has become an active research topic in wireless communications [5]-[6]. Meanwhile, beamforming (BF) technology can enhance the received signal power at legitimate users (LU) and suppress the signal leaked to the unintended users, and therefore is an effective method to improve the security performance of wireless systems. Nowadays, the BF-based PLS technology has been widely investigated in the terrestrial cellular networks [7]-[8] and SatCom networks [3],[9]-[10]. Specifically, the application of PLS in SatCom systems was studied in [9], where a sub-optimal $\mathrm{BF}$ algorithm based on zero forcing and an optimal BF method using semi-definite programming relaxation (SDR) were proposed, respectively. In [10], the authors proposed a BF algorithm to improve the security performance of SatCom through green source of terrestrial networks. All the previous works [9]-[10] verified that the PLS technology based on BF can effectively improve the security performance of the SatCom systems. However, the perfect channel state information (CSI) of eavesdroppers (Eves) is assumed to be known at the transmitter. In actual, this assumption does not hold since Eves are usually not authorized users [11]. Assuming that only the imperfect CSI of the wiretap channels is available, the authors in [3] proposed a robust BF scheme to maximize the minimal achievable secrecy rate (ASR) of LU under the constraint of total transmit power on the satellite. However, it should be mentioned that the work in [3] only focused on the scenario of a single Eve, which is only a special case. Although the PLS problem could be solved by converting it to the form of SDR or second-order cone program (SOCP), there is still an urgent demand for developing computationally more efficient algorithms. This observation motivates our work in this paper.

Unlike most of the existing works, we here consider a practical scenario where only the approximate locations of the Eves are available, and propose a robust BF scheme to enhance the security for multibeam satellite systems in the presence of Eves regardless of whether they are coordinated Eves (CE) or uncoordinated Eves (UE). We first formulate a constrained optimization problem to maximize the worst-case ASR of the LU, while satisfying the on-board per-antenna transmit power constraint and the quality of service (QoS) requirement for LU. Since the objective function is non-convex, we adopt the discretization method to deal with the unknown regions of the Eves and then approximate it through the log-sum-exp function. Afterwards,

J. Zhang, M. Lin and J. Ouyang are with the College of Telecommunications and Information Engineering, Nanjing University of Posts and Telecommunications, Nanjing, China.

W. -P. Zhu is with the Department of Electrical and Computer Engineering, Concordia University, Montreal, Canada, and also with the College of Telecommunications and Information Engineering, Nanjing University of Posts and Telecommunications, Nanjing, China.

T. de Cola is with the Institute of Communications and Navigation, German Aerospace Center (DLR), 82234 Oberpfaffenhofen, Germany.

Corresponding author: Min Lin (linmin@ njupt.edu.cn).

This work was supported in part by the Key International Cooperation Research Project under Grant 61720106003, in part by the National Natural Science Foundation of China under Grant 61801234, in part by the Shanghai Aerospace Science and Technology Innovation Foundation under Grant SAST2019-095, and in part by NUPTSF under Grant NY220111. 
an iteration algorithm joint Dinkelbach and Alternating Direction Method of Multipliers (ADMM) is adopted for solving this non-convex optimization problem. Compared to the existing approaches, the proposed scheme is computationally more efficient as each iteration step comes up with a simple closed-form solution.

Notations: Bold uppercase and lowercase letters denote matrices and vectors, respectively. $(\cdot)^{H}$ stands for the Hermitian transpose, $C^{m \times n}$ denotes the complex space of $m \times n, C N\left(\mu, \sigma^{2}\right)$ represents a complex Gaussian distribution with mean $\mu$ and covariance $\sigma^{2}$ matrix, $\mathbf{a} \odot \mathbf{b}$ denotes Hadamard product of two vectors a and $\mathbf{b},[x, 0]^{+}=\max \{x, 0\},[\mathbf{w}]_{n}$ represents the $n$-th component of $\mathbf{w},|\cdot|$ and $\|\cdot\|$ denote absolute value and Euclidean norm of a vector.

\section{SYSTEM MODEL}

In this paper, we consider the PLS for the downlink transmission of a multibeam satellite system, where a multibeam geostationary orbit (GEO) satellite sends private signal to the LU, while $K$ Eves within the satellite coverage attempt to overhear the private signal. Here, the GEO satellite configure $N$ antennas to generate $M$ beams. Besides, we suppose that only the imperfect CSI of each Eves is available ${ }^{1}$, which is a more practical assumption than that has been taken in the related works [9]-[10], where perfect CSI is exploited to carry out BF design for PLS.

Suppose that the multibeam satellite transmits signal $x(t)$ with normalized power to the LU after performing BF with weight vector $\mathbf{w} \in C^{N \times 1}$. The received signals at the LU and $k$-th Eve are, respectively, given by

$$
\begin{gathered}
\mathbf{y}_{s}=\mathbf{h}_{s}^{H} \mathbf{w} x(t)+n_{s}(t), \\
\mathbf{y}_{k}=\mathbf{h}_{k}^{H} \mathbf{w} x(t)+n_{k}(t), k=1 \ldots K,
\end{gathered}
$$

where $n_{s}(t)$ and $n_{k}(t)$ represent additive Gaussian white noises (AWGN) with zero means and the variances $\sigma_{i}^{2}=\kappa B_{i} T_{i}$, $i \in\{s, k\}$ at LU and $k$-th Eve respectively, with $\kappa=1.38 \times 10^{-23} \mathrm{~J} / K$ denoting Boltzmann constant, $T_{i}$ the noise temperature and $B_{i}$ the noise bandwidth. Further, $\left\{\mathbf{h}_{s}, \mathbf{h}_{k}\right\} \in \mathbb{C}^{N \times 1}$ are the channel vector between satellite and the LU, and that between satellite and $k$-th Eve, respectively. The channel vector of the satellite downlink can be written as [10]

$$
\mathbf{h}_{m}=\sqrt{G_{r}} \odot \mathbf{r}_{m}^{-\frac{1}{2}} \odot \mathbf{b}_{m}^{\frac{1}{2}} \odot \hat{\mathbf{h}}_{m}
$$

where $\mathbf{r}_{m}=\left[r_{m 1}, r_{m 2}, \ldots, r_{m N}\right]^{T}$ represents a rain attenuation fading vector, and whose entries expressed in $\mathrm{dB} r_{m n}^{\mathrm{dB}}=20 \log _{10}\left(r_{m n}\right)$ follow a lognormal random distribution, with $\mu$ and $\sigma_{r}$ being the lognormal location and the scale parameter, respectively. Besides $\mathbf{b}_{m}=\left[b_{m 1}, b_{m 2}, \ldots, b_{m N}\right]^{T}$ is the $N \times 1$ beam gain vector, whose component $b_{m n}$ is expressed as

$$
b_{m n}=b_{\max }\left(\frac{J_{1}\left(u_{m n}\right)}{2 u_{m n}}+36 \frac{J_{3}\left(u_{m n}\right)}{u_{m n}^{3}}\right)^{2},
$$

where $b_{\max }$ represents the maximal beam gain, $J_{1}(\cdot)$ and $J_{3}(\cdot)$ are the first-kind Bessel function of order 1 and 3 , respectively, and $u_{m n}=2.07123 \sin \phi_{m n} / \sin \phi_{3 \mathrm{~dB}}$ with $\phi_{m n}$ being the angle between the $m$-th user's position and the $n$-th beam center with respect to the satellite, and $\phi_{3 \mathrm{~dB}}$ the half power beamwidth. In (3), $\hat{\mathbf{h}}_{m}=\left[\hat{h}_{m 1}, \hat{h}_{m 2}, \ldots, \hat{h}_{m N}\right]^{T}$ is the channel response vector whose elements can be written as

$$
\hat{h}_{m n}=\frac{c}{4 \pi f_{c} d_{m n}} e^{-j \frac{2 \pi f_{c}}{c} d_{m n}}
$$

where $f_{c}$ denotes the carrier frequency and $c$ the light speed. The variable $d_{m n}$ is the distance between the $m$-th user and the $n$-th satellite antenna. In addition, let $G_{r}$ be the antenna gain of the user, as defined by [12]

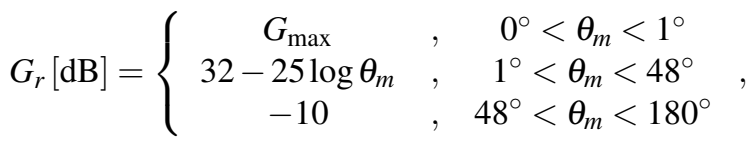

where $G_{\max }$ denotes the maximum antenna gain of the user, and $\theta_{m}$ is off-boresight angle. According to (1) and (2), the signal-to-noise ratio (SNR) of the LU and that of the $k$-th Eve are $\gamma_{s}=\left|\mathbf{h}_{s}^{H} \mathbf{w}\right|^{2} / \sigma_{s}^{2}$ and $\gamma_{k}=\left|\mathbf{h}_{k}^{H} \mathbf{w}\right|^{2} / \sigma_{k}^{2}$, respectively. Similar to [11], we consider two cases where $K$ Eves overhear the private signal uncooperatively and cooperatively, respectively. In the case of UE, the ASR of LU can be written as

$$
R_{s}=\min _{k \in\{1, \ldots, K\}}\left[\left(\log _{2}\left(1+\gamma_{s}\right)-\log _{2}\left(1+\gamma_{k}\right)\right), 0\right]^{+} .
$$

On the other hand, the ASR of LU in the case of CE can be expressed as

$$
R_{s}=\left[\left(\log _{2}\left(1+\gamma_{s}\right)-\log _{2}\left(1+\sum_{k=1}^{K} \gamma_{k}\right)\right), 0\right]^{+} \text {. }
$$

\footnotetext{
${ }^{1}$ In secure communications, the Eve is usually passive and keeps silent, and thus only imperfect CSI of Eve can be obtained. In satellite communications, the transmitter can estimate the channel from the approximate location of the Eve which can be obtained by satellite GPS [11].
} 
To guarantee the PLS in SatCom systems, we aim at designing the BF weight vector to maximize the ASR of the LU, which makes the ASR positive, and therefore the superscript "+" can be removed [9].

\section{Proposed BeAmformig Scheme}

In this paper, we take the CSI uncertainty of Eve into account, and propose a worst-case robust BF scheme to improve the security performance of the system, by considering the scenarios of UE and CE, respectively. We here consider that the satellite only knows the approximate locations of the Eves. In other words, each Eve is located in an approximate bounded by a rectangle and thus the CSI of the Eve can be expressed as

$$
\Psi_{k}=\left\{\mathbf{h}_{k} \mid x_{k} \in\left[x_{k, L}, x_{k, U}\right], y_{k} \in\left[y_{k, L}, y_{k, U}\right]\right\},\{k \in 1, \cdots, K\},
$$

where $x_{k, L}$ and $x_{k, U}$ denote the lower and upper bounds of the x-coordinate of the $k$-th Eve, and $y_{k, L}$ and $y_{k, U}$ the lower and upper bounds of the y-coordinate.

\section{A. Uncooperative Eves}

In this subsection, we focus on the case of UE, according (7) and (9), the worst-case problem can be written as

$$
\begin{gathered}
\max _{\mathbf{w}} \min _{k} \min _{\mathbf{h}_{k} \in \Psi_{k}} \log _{2}\left(1+\frac{\left|\mathbf{h}_{s}^{H} \mathbf{w}\right|^{2}}{\sigma_{s}^{2}}\right)-\log _{2}\left(1+\frac{\left|\mathbf{h}_{k}^{H} \mathbf{w}\right|^{2}}{\sigma_{k}^{2}}\right) \\
\text { s.t. } \frac{\left|\mathbf{h}_{s}^{H} \mathbf{w}\right|^{2}}{\sigma_{s}^{2}} \geqslant \gamma_{t h} \\
\left|[\mathbf{w}]_{n}\right|=\sqrt{p}, n=1, \ldots, N
\end{gathered}
$$

where $p$ represents the transmit power of each antenna, and $\gamma_{t h}$ the QoS requirement for LU. Here, we assume that the transmit power of per-antenna is constant, which can be easily implemented with low-cost phase shifters [13].

By denoting $\tilde{\mathbf{h}}_{s}=\mathbf{h}_{s} / \sigma_{s}, \tilde{\mathbf{h}}_{k}=\mathbf{h}_{k} / \sigma_{k}$ and considering that $\log _{2}(\cdot)$ is a monotonically increasing function, we can obtain an equivalent form of (10) as given below

$$
\begin{aligned}
\max _{\mathbf{w}} \min _{k} \min _{\mathbf{h}_{k} \in \Psi_{k}} \frac{1+\mathbf{w}^{H} \tilde{\mathbf{h}}_{s} \tilde{\mathbf{h}}_{s}^{H} \mathbf{w}}{1+\mathbf{w}^{H} \tilde{\mathbf{h}}_{k} \tilde{\mathbf{h}}_{k}^{H} \mathbf{w}} \\
\text { s.t. } \quad \mathbf{w}^{H} \tilde{\mathbf{h}}_{s} \tilde{\mathbf{h}}_{s}^{H} \mathbf{w} \geqslant \gamma_{t h} \\
\\
\quad\left|[\mathbf{w}]_{n}\right|=\sqrt{p}, n=1, \ldots, N .
\end{aligned}
$$

It can be observed that the optimization problem in (11) is equivalent to the following alternative form

$$
\begin{aligned}
& \min _{\mathbf{w}} \frac{\max _{k} \max _{\mathbf{h}_{k} \in \Psi_{k}}\left(1+\mathbf{w}^{H} \tilde{\mathbf{h}}_{k} \tilde{\mathbf{h}}_{k}^{H} \mathbf{w}\right)}{1+\mathbf{w}^{H} \tilde{\mathbf{h}}_{s} \tilde{\mathbf{h}}_{s}^{H} \mathbf{w}} . \\
& \text { s.t. (11b), (11c) }
\end{aligned}
$$

It is clear from (9) that the unknown area of $k$-th Eve $\Psi_{k}$ is a continuous rectangular area which makes the original problem in (12) nonconvex and mathematically intractable. To overcome this difficulty, we discretize the continuous region as

$$
\begin{gathered}
x_{k}^{(i)}=x_{k, L}+i \Delta x, i=0, \ldots, M_{1}-1, \\
y_{k}^{(i)}=y_{k, L}+j \Delta y, j=0, \ldots, M_{2}-1,
\end{gathered}
$$

where $\Delta x=\left(x_{k, U}-x_{k, L}\right) / M_{1}, \Delta y=\left(y_{k, U}-y_{k, L}\right) / M_{2}$. Suppose that the wiretap channel of the $k$-th Eve $\mathbf{h}_{k}$ belongs to a given discrete region as described below

At this point, we can obtain

$$
\begin{aligned}
\Lambda_{k}= & \left\{\mathbf{h}_{k}^{(i, j)} \mid x_{k}^{(i)}=x_{k, L}+i \Delta x, i=0, \ldots, M_{1}-1,\right. \\
& \left.y_{k}^{(j)}=y_{k, L}+j \Delta y, j=0, \ldots, M_{2}-1, k \in\{1, \ldots, K\}\right\}
\end{aligned}
$$

$$
\max _{k} \max _{\mathbf{h}_{k} \in \Psi_{k}}\left(1+\mathbf{w}^{H} \tilde{\mathbf{h}}_{k} \tilde{\mathbf{h}}_{k}^{H} \mathbf{w}\right)=\max _{k} \max _{\mathbf{h}_{k} \in \Lambda_{k}}\left(1+\mathbf{w}^{H} \tilde{\mathbf{h}}_{k} \tilde{\mathbf{h}}_{k}^{H} \mathbf{w}\right)
$$

Since the objective function of the optimization problem in (12) is a form of min-max-max, to tackle this difficulty, we here exploit the log-sum-exp function to approximate the objective function. Hence, equation (16) can be approximated as [14]

$$
\begin{aligned}
& \max _{k} \max _{\mathbf{h}_{k} \in \Lambda_{k}}\left(1+\mathbf{w}^{H} \tilde{\mathbf{h}}_{k} \tilde{\mathbf{h}}_{k}^{H} \mathbf{w}\right) \\
& \approx \beta^{-1} \ln \left(\sum_{k=1}^{K} \sum_{i=0}^{M_{1}-1} \sum_{j=0}^{M_{2}-1} e^{\beta\left(1+\mathbf{w}^{H} \tilde{\mathbf{h}}_{k}^{(i, j)} \tilde{\mathbf{h}}_{k}^{(i, j)}{ }^{H} \mathbf{w}\right)}\right)
\end{aligned}
$$


where $\beta$ is a parameter that regulates the degree of approximation, with the increase of $\beta$, the approximation error will gradually decrease. Substituting (17) into the optimization problem (12) and it can be reformulated as

$$
\min _{\mathbf{w}} \frac{\beta^{-1} \ln \left(\sum_{k=1}^{K} \sum_{i=0}^{M_{1}-1} \sum_{j=0}^{M_{2}-1} e^{\beta\left(1+\mathbf{w}^{H} \tilde{\mathbf{h}}_{k}^{(i, j)} \tilde{\mathbf{h}}_{k}^{(i, j)^{H}} \mathbf{w}\right)}\right)}{1+\mathbf{w}^{H} \tilde{\mathbf{h}}_{s} \tilde{\mathbf{h}}_{s}^{H} \mathbf{w}} .
$$

Obviously, the objective function in (18) is non-convex and in the form of fraction which can be efficiently solved by means of the Dinkelbach method [15]. The corresponding Dinkelbach sub-problem can then be written as

$$
\begin{aligned}
& \min _{\mathbf{w}} \Gamma(\mathbf{w})=\beta^{-1} \ln \sum_{k=1}^{K} \sum_{i=0}^{M_{1}-1} \sum_{j=0}^{M_{2}-1} e^{\beta\left(1+\mathbf{w}^{H} \tilde{\mathbf{h}}_{k}^{(i, j)} \mathbf{h}_{k}^{(i, j)}{ }^{H} \mathbf{w}\right)}-\eta\left(1+\mathbf{w}^{H} \tilde{\mathbf{h}}_{s} \tilde{\mathbf{h}}_{s}^{H} \mathbf{w}\right) . \\
& \text { s.t. (11b), (11c) }
\end{aligned}
$$

Since the problem in (19) is still non-convex and difficult to solve, we here exploit a non-convex ADMM algorithm to solve it. First, variables $\tilde{\mathbf{w}}$ and $\mathbf{x}$ are introduced to transform the above optimization problem to a suitable form for the ADMM solution framework, the problem in (19) is equivalent to the following alternative form

$$
\begin{aligned}
\min _{\tilde{\mathbf{w}}, \mathbf{x}} & \Gamma(\tilde{\mathbf{w}}) \\
\text { s.t. } & \tilde{\mathbf{w}}^{H} \tilde{\mathbf{h}}_{s} \tilde{\mathbf{h}}_{s}^{H} \tilde{\mathbf{w}} \geqslant \gamma_{t h}, \\
& \left|[\mathbf{x}]_{n}\right|=\sqrt{p}, n=1, \ldots, N, \\
& \mathbf{x}=\tilde{\mathbf{w}}
\end{aligned}
$$

Then, the augmented Lagrangian of (20) can be written as

$$
L(\tilde{\mathbf{w}}, \mathbf{x}, \mathbf{v})=\Gamma(\tilde{\mathbf{w}})+\operatorname{Re}\left\{\mathbf{v}^{H}(\tilde{\mathbf{w}}-\mathbf{x})\right\}+\frac{\rho}{2}\|\tilde{\mathbf{w}}-\mathbf{x}\|^{2}
$$

Here, $\mathbf{v}$ is a vector of Langrangian multiplier and $\rho$ the penalty factor. Finally, the variables $\{\tilde{\mathbf{w}}, \mathbf{x}, \mathbf{v}\}$ are cyclically updated according to the non-convex ADMM algorithm framework. Specifically, the following three steps are cycled through the solution process

$$
\left\{\begin{aligned}
\mathbf{x}^{(l+1)}= & \underset{\mid[\mathbf{x}]_{n} \models \sqrt{p}}{\arg \min } \operatorname{Re}\left\{\mathbf{v}^{(l)^{H}}\left(\tilde{\mathbf{w}}^{(l)}-\mathbf{x}\right)\right\}+\frac{\rho}{2}\left\|\tilde{\mathbf{w}}^{(l)}-\mathbf{x}\right\|^{2}, \\
\tilde{\mathbf{w}}^{(l+1)}= & \underset{\tilde{\mathbf{w}}^{H} \tilde{\mathbf{h}}_{s} \tilde{\mathbf{h}}_{s}^{H} \tilde{\mathbf{w}} \geq \gamma_{t h}}{\arg \operatorname{Re}}\left\{\nabla \Gamma\left(\mathbf{x}^{(l+1)}\right)^{H}\left(\tilde{\mathbf{w}}-\mathbf{x}^{(l+1)}\right)\right\} \\
& +\operatorname{Re}\left\{\mathbf{v}^{(l)}\left(\tilde{\mathbf{w}}-\mathbf{x}^{(l+1)}\right)\right\}+\frac{\rho+L}{2}\left\|\tilde{\mathbf{w}}-\mathbf{x}^{(l+1)}\right\|^{2}, \\
\mathbf{v}^{(l+1)}= & \mathbf{v}^{(l)}+\rho\left(\tilde{\mathbf{w}}^{(l+1)}-\mathbf{x}^{(l+1)}\right) .
\end{aligned}\right.
$$

In (23), $L$ is a constant greater than zero and satisfies $\left\|\nabla \Gamma\left(\mathbf{x}_{1}\right)-\nabla \Gamma\left(\mathbf{x}_{2}\right)\right\| \leq L\left\|\mathbf{x}_{1}-\mathbf{x}_{2}\right\|, \forall \mathbf{x}_{1}, \mathbf{x}_{2}$.

Next, let us elaborate on the solutions of ADMM sub-problems (22) and (23).

1) Update the variable $\mathbf{x}$

After some trivial computations, problem (22) can be rewritten as

$$
\begin{aligned}
\min _{\mathbf{x}} & \left\|\mathbf{x}-\left(\tilde{\mathbf{w}}^{(l)}+\rho^{-1} \mathbf{v}^{(l)}\right)\right\|^{2} . \\
\text { s.t. } & \left|[\mathbf{x}]_{n}\right|=\sqrt{p}, n=1, \ldots, N
\end{aligned}
$$

It is clear that problem (25) can be viewed as the Euclidean projection onto an Euclidean ball. The optimal solution is thus given by

$$
\left[\mathbf{x}^{(l+1)}\right]_{n}=\left\{\begin{array}{l}
\frac{\sqrt{p}\left[\tilde{\mathbf{w}}^{(l)}+\rho^{-1} \mathbf{v}^{(l)}\right]_{n}}{\left|\left[\tilde{\mathbf{w}}^{(l)}+\rho^{-1} \mathbf{v}^{(l)}\right]_{n}\right|},\left[\mathbf{w}^{(l)}+\rho^{-1} \mathbf{v}^{(l)}\right]_{n} \neq 0, \\
{\left[\mathbf{x}^{(l)}\right]_{n}, \text { else }}
\end{array}\right.
$$

2) Update the variable $\tilde{\mathbf{w}}$ 
Problem (23) can be rewritten as

$$
\begin{aligned}
& \min _{\tilde{\mathbf{w}}}\left\|\tilde{\mathbf{w}}-\left(\mathbf{x}^{(l+1)}-\frac{\nabla \Gamma\left(\mathbf{x}^{(l+1)}\right)+\mathbf{v}^{(l)}}{\rho+L}\right)\right\|^{2} . \\
& \text { s.t. } \tilde{\mathbf{w}}^{H} \tilde{\mathbf{h}}_{s} \tilde{\mathbf{h}}_{s}^{H} \tilde{\mathbf{w}} \geqslant \gamma_{t h}
\end{aligned}
$$

It can be observed that (27) defines a Quadratical Constraint Quadratic Programming problem with only one constraint, namely QCQP-1, which has a closed-form solution as discussed in [16].

Finally, the proposed algorithm is summarized as algorithm 1. The solution obtained by this algorithm is suboptimal, but the optimal solution can be obtained by arbitrarily reducing the iteration parameter $\varepsilon$. The major complexity comes from ADMM iterations. And the total computational complexity of each ADMM iteration is $O\left(K M_{1} M_{2}\left(8 N^{2}+11 N+5\right)+16 N^{2}+27 N\right)$.

\section{B. Cooperative Eves}

In this subsection, we extend the proposed algorithm to the case of CE. In this regard, the constrained optimization problem can be formulated as the following worst-case problem

$$
\begin{gathered}
\max _{\mathbf{w}} \min _{\mathbf{h}_{k} \in \Psi_{k}} \log _{2}\left(1+\frac{\left|\mathbf{h}_{s}^{H} \mathbf{w}\right|^{2}}{\sigma_{s}^{2}}\right)-\log _{2}\left(1+\sum_{k=1}^{K}\left(\frac{\left|\mathbf{h}_{k}^{H} \mathbf{w}\right|^{2}}{\sigma_{k}^{2}}\right)\right) \\
\text { s.t. } \frac{\left|\mathbf{h}_{s}^{H} \mathbf{w}\right|^{2}}{\sigma_{s}^{2}} \geqslant \gamma_{t h} \\
\left|[\mathbf{w}]_{n}\right|=\sqrt{p}, n=1, \ldots, N
\end{gathered}
$$

Similar to (10), the original problem in (28) can be written as

$$
\begin{aligned}
\max _{\mathbf{w}} \min _{\mathbf{h}_{k} \in \Psi_{k}} \frac{1+\mathbf{w}^{H} \tilde{\mathbf{h}}_{s} \tilde{\mathbf{h}}_{s}^{H} \mathbf{w}}{1+\sum_{k=1}^{K}\left(\mathbf{w}^{H} \tilde{\mathbf{h}}_{k} \tilde{\mathbf{h}}_{k}^{H} \mathbf{w}\right) .} \\
\text { s.t. }(11 b),(11 c)
\end{aligned}
$$

Further, the optimization problem in (29) is equivalent to the following alternative form

$$
\begin{aligned}
& \min _{\mathbf{w}} \frac{1+\sum_{k=1}^{K}\left(\max _{\mathbf{h}_{k} \in \Psi_{k}}\left(\mathbf{w}^{H} \tilde{\mathbf{h}}_{k} \tilde{\mathbf{h}}_{k}^{H} \mathbf{w}\right)\right)}{1+\mathbf{w}^{H} \tilde{\mathbf{h}}_{s} \tilde{\mathbf{h}}_{s}^{H} \mathbf{w}} . \\
& \text { s.t. }(11 b),(11 c)
\end{aligned}
$$

With the help of log-sum-exp function, we can obtain

$$
\begin{aligned}
\max _{\mathbf{h}_{k} \in \Psi_{k}} \mathbf{w}^{H} \tilde{\mathbf{h}}_{k} \tilde{\mathbf{h}}_{k}^{H} \mathbf{w} & =\max _{\mathbf{h}_{k} \in \Lambda_{k}} \mathbf{w}^{H} \tilde{\mathbf{h}}_{k} \tilde{\mathbf{h}}_{k}^{H} \mathbf{w} \\
& \approx \beta^{-1} \ln \left(\sum_{i=0}^{M_{1}-1} \sum_{j=0}^{M_{2}-1} e^{\beta\left(\mathbf{w}^{H} \tilde{\mathbf{h}}_{k}^{(i, j)} \tilde{\mathbf{h}}_{k}^{(i, j)}{ }^{H} \mathbf{w}\right.}\right)
\end{aligned}
$$

Therefore, the optimization problem (30) can be converted to

$$
\min _{\mathbf{w}} \frac{1+\sum_{k=1}^{K}\left(\beta^{-1} \ln \left(\sum_{i=0}^{M_{1}-1} \sum_{j=0}^{M_{2}-1} e^{\beta\left(\mathbf{w}^{H} \tilde{\mathbf{h}}_{k}^{(i, j)} \tilde{\mathbf{h}}_{k}^{(i, j)}{ }^{H} \mathbf{w}\right)}\right)\right)}{1+\mathbf{w}^{H} \tilde{\mathbf{h}}_{s} \tilde{\mathbf{h}}_{s}^{H} \mathbf{w}} .
$$

Similar to the optimization problem in (18), the objective function of this problem is non-convex and in the form of fractions, which can still be solved by the Dinkelbach method. The corresponding sub-problem is given by

$$
\begin{aligned}
& \min _{\mathbf{w}} 1+\sum_{k=1}^{K}\left(\beta^{-1} \ln \left(\sum_{i=0}^{M_{1}-1} \sum_{j=0}^{M_{2}-1} e^{\beta\left(\mathbf{w}^{H} \tilde{\mathbf{h}}_{k}^{(i, j)} \tilde{\mathbf{h}}_{k}^{(i, j)}{ }^{H} \mathbf{w}\right.}\right)\right) \\
& -\eta\left(1+\mathbf{w}^{H} \tilde{\mathbf{h}}_{s} \tilde{\mathbf{h}}_{s}^{H} \mathbf{w}\right) \\
& \text { s.t. }(11 b),(11 c)
\end{aligned}
$$

The optimization problem in (33) has the same form as (19), and thus can be solved by the non-convex ADMM algorithm framework, which is omitted here due to space limitation. 


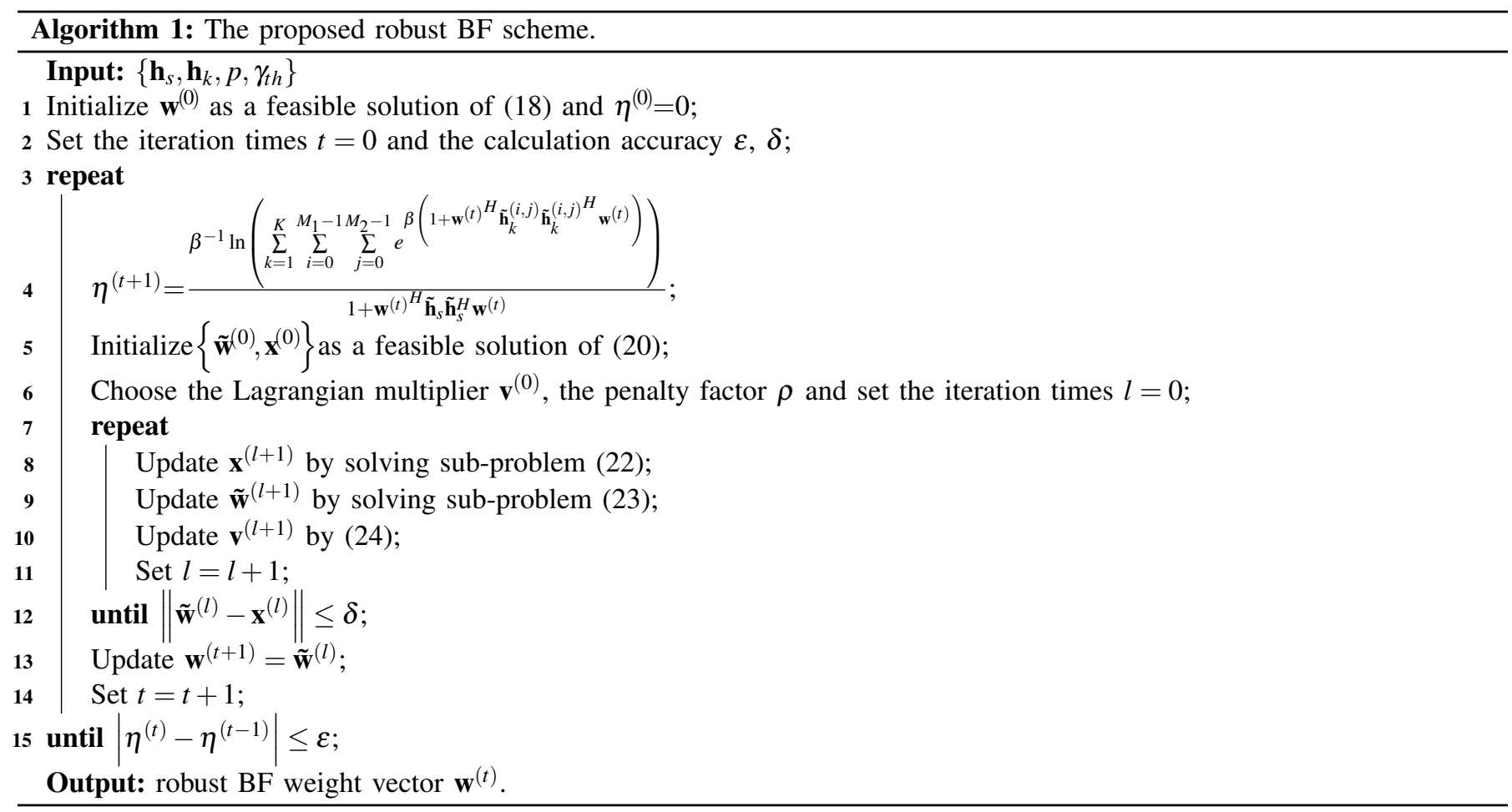

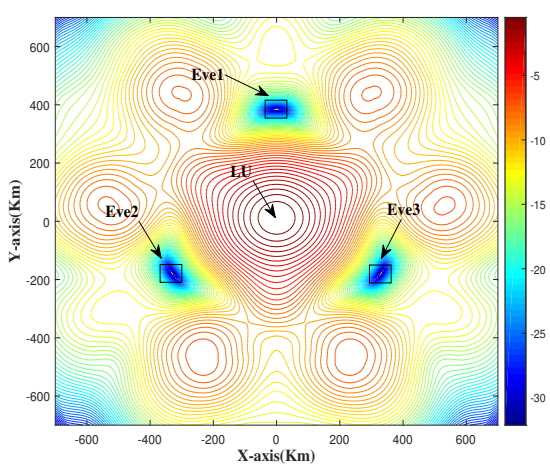

Fig. 1. Beampattern for UE

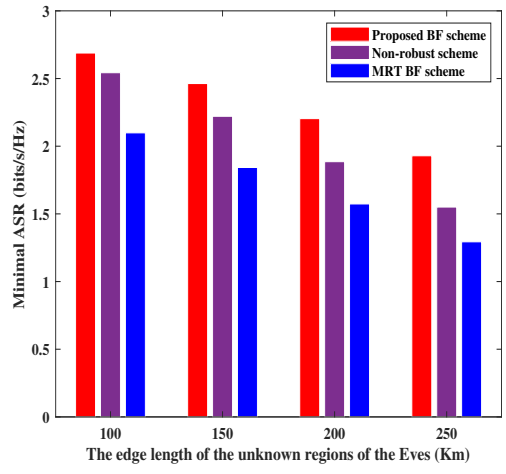

Fig. 2. Minimal ASR versus the edge length of the Fig. 3. Minimal ASR versus the power of perunknown regions of the Eves

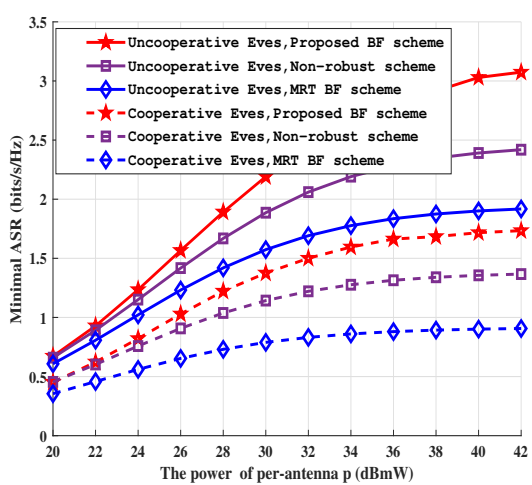
antenna

\section{NumericAl RESUltS}

In this section, we provide simulation results to verify the effectiveness and superiority of our proposed scheme. The MRT $\mathrm{BF}$ and the non-robust BF schemes are taken as benchmarks for the sake of comparison. Unless otherwise indicated, we set the number of beams $N=7$, the number of Eves $K=3$, the QoS threshold of LU $\gamma_{t h}=5$, the edge length of the uncertain area as 100 $\mathrm{Km}$, and the approximation parameter $\beta=100$. The other system parameters are set by referring to [3][17].

Fig. 1 presents the beampattern for the case of UE. It can be found that the maximal direction of beamforming points to LU, while three nulls are generated in the estimated region of Eves, respectively. It not only enhances the signal power of the LU but also inhibits the signal quality of the Eves.

The influence of the edge length of the unknown regions of the Eves on minimal ASR for the case of UE is depicted in Fig. 2. The per-antenna transmit power is assumed as $30 \mathrm{dBmW}$, it can be found that the minimum ASR of LU decreases as the edge length of the unknown regions of the Eves increases. Fig. 3 gives the minimal ASR versus the transmit power budget of the per-antenna for three BF schemes by assuming that the edge length of the unknown regions of the Eves is $200 \mathrm{Km}$. It can be observed that our proposed robust BF scheme is superior to the MRT BF scheme and the non-robust BF scheme in both cases of UE and CE. The reason is that the MRT scheme only focuses on the maximum SNR of the LU, without considering the ASR, and the non-robust BF scheme does not take the unknown regions of the Eves into consideration.

To confirm the computational advantage of the proposed algorithm, the SDR-based method is adopted for comparison. The CPU running times of the two approaches, with different numbers of Eves, are listed in Table I. It can be observed that the proposed algorithm runs much faster than the SDR method. 
Table I: Comparison of the CPU running times (seconds)

\begin{tabular}{|c|c|c|}
\hline Number of Eves & SDR CPU Time & Proposed CPU Time \\
\hline 1 & 1.012 & 0.0147 \\
\hline 2 & 1.406 & 0.0445 \\
\hline 3 & 1.911 & 0.0759 \\
\hline 4 & 2.517 & 0.1733 \\
\hline
\end{tabular}

\section{CONCLUSION}

This paper has investigated the PLS in the downlink of a multibeam satellite system. Specifically, we first formulated a optimization problem to maximize the worst-case ASR of the LU. Since the original problem is nonconvex and intractable, we adopted log-sum-exp function to approximate the objective function, and then exploited ADMM combined with Dinkelbach method to obtain the BF weight vector. Finally, simulation results have confirmed the effectiveness and superiority of the proposed robust BF scheme. In our future works, we will consider other effects, such as hardware impairments in BF design to enhance security in multibeam satellite systems.

\section{REFERENCES}

[1] A. I. Perez-Neira, M. A. Vazquez, M. R. B. Shankar, S. Maleki, and S. Chatzinotas, "Signal processing for high-throughput satellites: Challenges in new interference-limited scenarios," IEEE Signal Process. Mag., vol. 36, no. 4, pp. 112-131, 2019.

[2] C. Qi and X. Wang, "Precoding design for energy efficiency of multibeam satellite communications," IEEE Commun. Lett., vol. 22, no. 9, pp. 1826-1829, 2018.

[3] Z. Lin, M. Lin, J. Ouyang, W. P. Zhu, A. D. Panagopoulos, and M. S. Alouini, "Robust secure beamforming for multibeam satellite communication systems," IEEE Trans. Veh. Technol., vol. 68, no. 6, pp. 6202-6206, 2019.

[4] Q. Huang, M. Lin, W. P. Zhu, S. Chatzinotas, and M. S. Alouini, "Performance analysis of integrated satellite-terrestrial multiantenna relay networks with multiuser scheduling," IEEE Trans. Aerosp. Electron. Syst., vol. 56, no. 4, pp. 2718-2731, 2020.

[5] X. Chen, D. W. K. Ng, W. H. Gerstacker, and H. Chen, "A survey on multiple-antenna techniques for physical layer security," IEEE Commun. Surveys Tuts., vol. 19, no. 2, pp. 1027-1053, 2017.

[6] Z. Lin, M. Lin, B. Champagne, W. P. Zhu, and N. Al-Dhahir, "Secure and energy efficient transmission for RSMA-based cognitive satellite-terrestrial networks," IEEE Wireless Commun. Lett., vol. 10, no. 2, pp. 251-255, 2021.

[7] K. Cumanan, Z. Ding, M. Xu, and H. V. Poor, "Secrecy rate optimization for secure multicast communications," IEEE J. Sel. Topics Signal Process., vol. 10, no. 8, pp. 1417-1432, 2016.

[8] Z. Lin, M. Lin, B. Champagne, W. P. Zhu, and N. Al-Dhahir, "Secure beamforming for cognitive satellite terrestrial networks with unknown eavesdroppers," IEEE Syst. J., early access, Apr.2020, doi:10.1109/JSYST.2020.2983309.

[9] G. Zheng, P. Arapoglou, and B. Ottersten, "Physical layer security in multibeam satellite systems," IEEE Trans. Wireless Commun., vol. 11, no. 2, pp. 852-863, 2012.

[10] M. Lin, Z. Lin, W. P. Zhu, and J. Wang, "Joint beamforming for secure communication in cognitive satellite terrestrial networks," IEEE J. Sel. Areas Commun., vol. 36, no. 5, pp. 1017-1029, 2018.

[11] Z. Lin, M. Lin, J. Wang, Y. Huang, and W. P. Zhu, "Robust secure beamforming for 5G cellular networks coexisting with satellite networks," IEEE J. Sel. Areas Commun., vol. 36, no. 4, pp. 932-945, 2018.

[12] ITU-R Recommendation S.465, Reference radiation pattern for earth station antennas in the fixed-satellite service for use in coordination and interference assessment in the frequency range from 2 to $31 \mathrm{GHz}$, International Telecommunication Union, 2010.

[13] B. Ning and Z. Chen, "Low-complexity analog beamforming for mmwave large-scale MISOME wiretap channel," IEEE Commun. Lett., vol. 24, no. 2 , pp. $268-271,2020$.

[14] Y. Chen and D. Y. Gao, "Global solutions to nonconvex optimization of 4th-order polynomial and log-sum-exp functions," J. Global. Optim, vol. 64, no. 3, pp. 417-431, 2016.

[15] W. Dinkelbach, "On nonlinear fractional programming," Manage. Sci, vol. 13, no. 7, pp. 492-498, 1967.

[16] K. Huang and N. D. Sidiropoulos, "Consensus-ADMM for general quadratically constrained quadratic programming," IEEE Trans. Signal Process., vol. 64 , no. 20, pp. 5297-5310, 2016.

[17] Z. Lin, M. Lin, T. de Cola, J. B. Wang, W. P. Zhu, and J. Cheng, "Supporting IoT with rate-splitting multiple access in satellite and aerial integrated networks," IEEE Internet Things J., early access, Jan. 2021, doi: 10.1109/JIOT.2021.3051603. 